\title{
LA NEUROEDUCACIÓN Y EL TRASTORNO PERSONAL ANTISOCIAL (TPA)
}

\author{
David Parra Reyes
}

Katiuska del Águila Camargo

\begin{abstract}
RESUMEN
La educación peruana actual vive momentos muy críticos debido a que el proceso de aprendizaje se ve simplificado a la mera memorización o mecanización de conceptos $\mathrm{y} / 0$ procesamientos de datos que no contribuyen a un aprendizaje significativo y que más bien está influenciando al desinterés educativo por parte de los estudiantes. Es por ello que mediante este artículo se pretende despertar el interés de las personas responsables de la educación de los educandos y que mediante el conocimiento de las funciones cerebrales se logre elaborar las herramientas necesarias que contribuyan a desarrollar las capacidades que se debe de tener para el logro de los aprendizajes esperados.
\end{abstract}

Estudios actuales en neuroeducación han demostrado que muchos estudiantes han desarrollado trastornos personales antisociales por diversos factores que han contribuido para la aparición de estos, los cuales al mismo tiempo están generando mucha violencia y delincuencia juvenil.

\section{PALABRAS CLAVE}

Neurociencia, educación, neuroeducación, trastorno personal antisocial.

\section{ABSTRACT}

The current Peruvian education is experiencing very critical moments since the learning process looks simplified to the simple memorization or mechanization of concepts and/or data processing which do not contribute to significant learning and that is rather influencing on the educational disinterest for the students. Reason why the present article aims to awaken the interest of responsible people for the education of learners and that through the knowledge of brain functions can be developed the necessary tools that contribute to develop the capacities that must be for the achievement of expected learning.

Current studies in neuroeducation have shown that many students have developed personal antisocial disorders because of several factors that have contributed to the emergence of these, which at the same time are generating much violence and juvenile crime.

\section{KEYWORDS}

Neuroscience, education, neuroeducation, antisocial personal disorder.

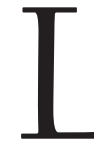

a época en que vivimos está marcada por la creciente búsqueda del desarrollo del potencial humano, el cual se relaciona con el complejo proceso de desarrollo y maduración del sistema nervioso centraly especialmente del cerebro en conjunción con las influencias del medio ambiente.

Para ello se ha echado mano de un conjunto de disciplinas que mediante el estudio del funcionamiento cerebral han logrado dar luces a muchas interrogantes que se tenía sobre el desenvolvimiento del ser humano y de cómo influye en su comportamiento y en la forma de vida que desarrolla; estas disciplinas son conocidas comoneurociencias.

Podría indicarse que las neurociencias nacen en el año 1600 a.C., aproximadamente, a través de la explicación de la psiquis, pasando por los aportes de las ciencias básicas, recorriendo la época del renacimiento y el desarrollo científico de la edad moderna hasta los anatomistas y microscopistas del siglo XX. Sin embargo, tenemos que resaltar que hasta hace apenas 30 años, se conocía muy poco sobre las funciones cerebrales específicas o especializadas.

Gracias a la neurociencia, se han abierto luces en el campo pedagógico por ejemplo, el cual ha generado revelaciones acerca de 
las bases neurales del aprendizaje, de la memoria, de las emociones y de muchas otras funciones cerebrales que son estimuladas y fortalecidas en el aula diariamente. Por ello debe ser un requisito importante que todo docente conozca y entienda cómo aprende el cerebro, cómo procesa la información que recibe, cómo controla las emociones, los sentimientos, los estados conductuales, o cómo es frágil frente a determinados estímulos, para la innovación pedagógica y transformación de los sistemas educativos. En este sentido, es la neuroeducación (área de la neurociencia aplicada en la educación con apoyo de la psicología) quien contribuye a disminuir la brecha entre las investigaciones neurocientíficas y la práctica pedagógica.

\section{LOS APORTES DE LA NEUROEDUCACIÓN EN ELAPRENDIZAJE SIGNIFICATIVO}

El campo educativo tiene como objetivo principal lograr el desarrollo máximo del potencial de las habilidades cognitivas y ejecutivas de los estudiantes, así como su empatía; esto solo se va a lograr mediante el conocimiento de las funciones cerebrales por parte de los docentes y facilitadores.

Si afirmamos esto es porque actualmente la educación vive momentos preocupantes, debido que en la mayoría de los casos, ésta se basa solamente en prácticas memorísticas que no contribuyen a un verdadero aprendizaje.

A lo largo del desarrollo de las sociedades, han aparecido diversas teorías que explicaban la adquisición del conocimiento, con el paso del tiempo estas ideas han ido evolucionando debido a la aparición de nuevos conocimientos y el surgimiento de nuevas necesidades de las sociedades.

Según la división histórica de Carlos Marx al remontándonos a la época del esclavismo, aparece en la antigua Grecia el concepto de aprender, el cual significaba la "modificación del comportamiento". Allí la comunidad y el individuo eran responsables unos de otros, generándose de esta manera la transformación, integración y desarrollo de las sociedades; el individuo era percibido desde diferentes aspectos y conformaba parte de una cadena social, cada miembro de esta sociedad era importante pues contribuía para la evolución de su contexto social.

Posteriormente durante la edad moderna o mercantilista (S. XV - XVII), se abandona esta conceptualización y se valora al individuo por lo que producía, quien acumulaba la mayor cantidad de conocimientos, era quien más destacaba, los otros individuos de esta sociedad no tenían mayor participación y eran considerados como un grupo social poco apreciado; sin embargo, hay que tener en cuenta que no todos tenían el privilegio de poder acudir a una escuela.

En la década de los 80, la educación en masa forma parte del escenario educacional y a través de esa mayor diversidad, se pudo percibir que el sistema educacional necesitaba de un gran cambio, todo lo que se manejaba sobre educación, aprendizaje y comportamiento humano era el fruto de investigaciones anteriores al escaneo cerebral.

Gracias a los estudios del cerebro, científicos y educadores que son conocedores del funcionamiento del cerebro, pretenden aplicar estos conocimientos con el propósito de ayudar a aprender y, sobre todo, de enseñar mejor.

El Dr. Carlos Logatt Grabner, médico oncólogo de la Universidad de Buenos Aires, Magíster en Neurociencia y Biología del Comportamiento, Universidad de Murcia y actual Presidente de la Asociación Educar, es el responsable de la creación y desarrollo de la neuroeducación. Sus aportes comenzaron hace más de 21 años como parte del proyecto Línea de Cambio, cuya finalidad está orientada a la comprensión y mejoramiento de la conducta humana.

A través de la Asociación Educa, el Dr. Logatt en el 2014, expresa que "La capacidad de estudio en cualquier edad se encuentra íntimamente relacionada con un factor primordial: la atención selectiva, que se ve acrecentada, principalmente, por la curiosidad o el entusiasmo que nos genere un aprendizaje. Es realmente importante encontrarle un sentido a lo que estudiamos, y que no sólo sea el simple hecho de rendir un examen". 
Es así que con la llegada de la neuroeducación se comienza a experimentar ciertos cambios en la forma de abordar el proceso formativo educacional.

Por tal motivo, la neuroeducación ha traído consigo abordajes diferenciados para el contexto educacional, los cuales indican que es necesario volver a los orígenes de la antigua Grecia y apreciar a al ser humano como un ser integral, global, no individual, ya que todas las áreas "especializadas" en él, en realidad actúan de manera interdisciplinaria.

Pese a que el término neuroeducación no es muy conocido aun en nuestro medio, debemos aclarar que no es una nueva área del conocimiento ya que se trata de la unión de los conocimientos de la psicología (considerada como el área que auxilia a la educación), de la educación y de la neurociencia.

La neuroeducación nos trae un abordaje diferenciado de lo que es el aprendizaje y la adquisición de nuevos conocimientos. Si el educando es evaluado por las características de los conocimientos adquiridos dentro del contexto escolar entonces podríamos decir que la educación no está siendo inclusiva, pero si el conocimiento que se percibe del educando ha modificado su comportamiento inicial en cualquiera de sus áreas (psicomotora, cognitiva o emocional), sí estaríamos hablando de una educación inclusiva, los cuales priman en los derechos humanos.

El neuroeducador rescata la práctica de la antigua Grecia, al observar al individuo en sus aspectos que precisan ser mejorados y en sus potencialidades, para ello debe de construir un planteamiento individualizado para cada educando.

\section{EL APRENDIZAJE: UN CONCEPTO ASOCIADO}

Conocer esos códigos de funcionamiento del cerebro ha permitido demostrar, por ejemplo, la importancia de la curiosidad y la emoción para adquirir conocimientos y que el deporte, la sorpresa y la experimentación también son algunos de los ingredientes necesarios para sumar nuevos conocimientos.

Además, estudios científicos realizados en la actualidad indican que la memorización y la repetición, no es la mejor herramienta para lograr un aprendizaje inclusivo.

En ese sentido, se podría indicar que la emoción es el ingrediente secreto del aprendizaje, y según la neuroeducación, es fundamental para quien enseña y para quien aprende. "El binomio emoción-cognición es indisoluble, intrínseco al diseño anatómico y funcional del cerebro", nos indica Francisco Mora, catedrático de Fisiología Humana (Universidad Complutense) y catedrático adscrito de Fisiología Molecular y Biofísica (Universidad de Iowa, EE.UU.), autor de Neuroculturayneuroeducación.

La neuroeducación ha demostrado que la información que captamos por medio de los sentidos debe atravesar por el sistema límbico antes de ser enviada a la corteza cerebral. Dentro del sistema límbico, la amígdala cerebral, que está localizada en la profundidad del lóbulo temporal y cuyo papel principal es el procesamiento y almacenamiento de reacciones emocionales, se activa ante eventos que considera importantes para la supervivencia, lo cual consolida un recuerdo de manera más eficiente. Las historias y relatos, suelen fungir como auténticos activadores de esta región cerebral. Por ejemplo, si en una clase de matemática toca explicar, el triángulo de Tartaglia, se puede explicar que en realidad el matemático italiano que lo formuló no se llamaba Tartaglia, sino Niccolò Fontana y que como era tartamudo, tartaglia, en italiano. Entonces ese mote acabó dando nombre a la fórmula. Esa anécdota hace estallar de risa a los estudiantes, y lo mejor es que ya no se olvidan de la fórmula.

Como vemos, las emociones mantienen la curiosidad y nos sirven para comunicarnos, por lo tanto es imprescindible en los procesos de razonamiento y toma de decisiones del ser humano, por lo tanto, los procesos cognitivos y los procesos emocionales son inseparables (Damasio, A. 1994). Posteriormente, Erk en el año 2003, indica que "las emociones positivas facilitan la 
memoria y el aprendizaje, mientras que en el estrés crónico la amígdala dificulta el paso de información del hipocampo a la corteza prefrontal, sede de las funciones ejecutivas".

La sorpresa es otro factor indispensable para la activación de la amígdala. Al cerebro le gusta procesar patrones, entender cosas que se repiten siempre de la misma forma. Todo aquello que no forma parte de esos patrones se guarda de manera más profunda en el cerebro. Es por ello que se debe usar en el aprendizaje, elementos que rompan con la monotonía, los cuales van a beneficiar el estudio.

La neuroeducación defiende la idea de la existencia de "ventanas", esto nos indica que el cerebro no es estático, sino que "existen ventanas plásticas, periodos críticos en los que un aprendizaje se ve más favorecido que otro". Por ejemplo, para aprender a hablar la "ventana" se abre al nacer y se cierra a los siete años, aproximadamente. Eso no quiere decir que pasada esa edad el niño no pueda adquirir el lenguaje, porque gracias a la plasticidad del cerebro que es la capacidad de las células nerviosas para regenerarse anatómica y funcionalmente, como consecuencia de estimulaciones ambientales, lo conseguirá aunque le cueste mucho más, no se podrá adquirir el dominio de la lengua que tiene un niño que aprendió a hablar de los 0 a los 3 años.

El hallazgo de la existencia de periodos de aprendizaje hace que las escuelas deban replantearse el modelo educativo, los estudios realizados nos indican que "hasta los 10 o 12 años, el cerebro tiene una ventana específica para aprender aptitudes, para manejar información, para razonar". Tal vez esa etapa sea el momento de potenciar la comprensión de un texto; que aprendan a razonar de forma matemática, en lugar de memorizar mucho contenido.

La neuroeducación aconseja que para construir buenas ideas hay que tener buenos preceptos. Son los átomos del conocimiento, del pensamiento; no podemos entender la educación si no tenemos en cuenta cómo funciona el cerebro.

Es por ello que sería recomendable que los docentes y facilitadores conozcan sobre el funcionamiento del cerebro para enseñar mejor y dejar el sistema anacrónico que se viene aplicando hasta hoy en día.

Resumiendo lo dicho anteriormente si entendemos la educación como un proceso de aprendizaje para la vida, la educación emocional resulta imprescindible porque contribuye al bienestar personal y social.

\section{LA NEUROEDUCACIÓN Y LOS TRASTOR- NOS PERSONALES ANTISOCIALES}

¿Se podría considerar que la educación actual influye en la personalidad de los educandos y que, a su vez, genera trastornos de personalidad antisocial?

Para ello primero debemos de indicar que la personalidad se define como una organización dinámica que se realiza entre los diferentes sistemas psicobiológicos del individuo el cual genera una mejor adaptación. Esta organización depende tanto de la maduración neurobiológica, como de las experiencias interpersonales y afectivas, además de la incorporación de normas sociales". Garzón, Á. M., \& Sánchez, J. A. (2007), indican que Pichot (1995) afirma que para generarse un trastorno de personalidad se necesita la implicancia de una serie de factores que deben tener un patrón permanente de experiencias internas y de comportamiento, las cuales aparta al sujeto de las expectativas culturales, las que están inmersas en una, dos o más áreas como por ejemplo la cognición, de la cual se interpreta a uno mismo, a los demás y a los acontecimientos; la afectividad, donde encontramos la intensidad, labilidad y la adecuación de la respuesta emocional, la actividad interpersonal y para finalizar el control de los impulsos.

El manual diagnóstico y estadístico de los trastornos mentales (DSM-IV), divide los trastornos de la personalidad en cuatro grupos: en el grupo A se encuentra la personalidad paranoide, la esquizoide y la esquizotípica. Las del grupo B incluyen los trastornos de la personalidad antisocial, borderline, histriónica y narcisista, teniendo así en las personas que las padecen comportamientos con inclinación al dramatismo, a la emotividad y a conductas erráticas. El grupo C comprende los trastornos de la personalidad por 
Tabla 1

Clasificación de los trastornos personal antisocial (TPA)

\begin{tabular}{|c|l|}
\hline Criterio & \multicolumn{1}{c|}{ Descripción } \\
\hline A & A1: No hay adaptación a las normas en torno al comportamiento legal. \\
& A2: Desprecian deseos, derechos y sentimientos de los demás. Engañan y \\
& manipulan para sacar provecho propio. \\
& A3: Incapacidad para planificar el futuro. \\
& A4: Irritables y agresivos (pelea física). \\
& A5: Despreocupación por la propia seguridad y la de los demás. \\
& A6: Continua y extremadamente irresponsables. \\
& A7: Tienen poco remordimiento por las consecuencias de sus actos. \\
\hline B & El individuo debe tener, por lo menos, dieciocho años. \\
\hline C & El individuo debe tener un historial de algunos síntomas de trastorno disocial de \\
& personalidad antes de los quince años. \\
\hline D & El comportamiento antisocial no debe aparecer exclusivamente en el transcurso \\
& de una esquizofrenia o de un episodio maníaco. \\
\hline
\end{tabular}

Fuente: DSM-IV

evitación, por dependencia y el obsesivocompulsivo, y una categoría denominada trastorno de la personalidad no especificado, en la cual las personas suelen ser ansiosas y temerosas.

En el 2003, Garrido afirma que "Es importante indicar que este trastorno se inicia con comportamientos sutiles en la niñez y se exhiben de modo intenso después de la adolescencia y durante la etapa de adulto joven, para luego disminuir cuando se llega a la edad de 40 años". Asimismo, este autor explica que el TPA presenta rasgos de personalidad compartidos o parecidos con otros trastornos de la personalidad, como:

Antisocial (solo): presentar historia de trastorno disocial (menor de quince años), impulsividad, agresividad, comportamiento delictivo y mentiras.

Narcisista (solo): donde la persona busca despertar admiración y envidia en los demás.

Antisocial-Narcisista: al igual que el antisocial, presenta historia de trastorno disocial (menor de quince años), i mpulsividad, agresividad, comportamiento delictivo, mentiras, más características como ser explotadores con poca sinceridad y empatía.
Antisocial-Histriónico: se observan conductas como la impulsividad, la superficialidad, imprudencia, manipulación y búsqueda de sensaciones.

Por consiguiente, ante la afirmación que existe una relación entre la educación actual deficiente con las conductas antisociales, podemos deducir que si existe, pero que al mismo tiempo observamos, que influyen significativamente la aparición de estas conductas antisociales en el transcurso de su vida.

\section{FACTORES QUE CONTRIBUYEN EN LA CONDUCTA Y ELAPRENDIZAJE}

Ante la presencia continua de estas conductas surge la necesidad de estudiar las estructuras cerebrales implicadas en los diferentes procesos de la psicopatía en donde la ciencia médica ha utilizado los avances técnicos de neuroimagen funcional, como la Tomografía por Emisión de Positrones (TEP), la Tomografía Computarizada por emisión de Fotón Único (SPECT) y la Resonancia Magnética Funcional (FMRI) para hallar relaciones entre las regiones cerebrales y los criterios diagnósticos del Trastorno Personal Antisocial(TPA).

Del-Ben, en 2005, indica que en los estudios se encontró que las personas diagnosticadas con 
TPA presentan una reducción en el volumen de la masa prefrontal del cerebro, el cual se correlaciona positivamente con una reducción en la respuesta autónoma frente a un evento estresor. Garzón, Á. M., \& Sánchez, J. A. (2007), citan a los investigadores Chiana-Shan, Kosten y Shinha (2006), quienes refieren que los individuos con TPA no tienen una adecuada respuesta emocional ante las situaciones de estrés y no logran aprender de las asociaciones cuando se enfrentan a situaciones similares.

En investigaciones posteriores se halló que las personas antisociales suprimen el dolor emocional ante estímulos lingüísticos y no lingüísticos agresivos, lo cual va correlacionado con una baja sensibilidad para llevar a cabo un proceso de aprendizaje significativo ante las señales de castigo. Por lo tanto, ante una situación de estrés se observa una disminución en la actividad del complejo amígdala-hipocampo, giro parahipocampal, estriado ventral y giro del cíngulo posterior y anterior del cerebro.

Se debe tomar en cuenta que además de los estudios que se han realizado sobre las estructuras anatómicas, también es importante resaltar los estudios sobre el funcionamiento hormonal de todo cuerpo, los cuales influyen en el desarrollo del TPA.

La discrepancia fisiológica del género nos indica que los hombres presentan mayor frecuencia de conducta antisocial en la infancia y en la adolescencia que las mujeres, en proporción de 4:1. En la edad adulta, los hombres muestran mayor prevalencia de trastorno de personalidad antisocial y conductas delictivas con relación de 7:1. La tipología del delito por género es diferente. Las mujeres cometen con mayor frecuencia delitos menores, mientras los hombres muestran mayor frecuencia de delitos contra la vida y la propiedad privada (APA, 1999). Mata en 1998, indica que las diferencias de género se deben a las hormonas gonadales, en particular la testosterona, y han sido asociadas con la sexualidad, la dominancia social y la agresividad en animales; es la testosterona la que parece estar relacionada con la dominancia social, la búsqueda de sensaciones (desinhibición) y experiencias heterosexuales.
Tomando en cuenta la influencia que ejerce no solo el funcionamiento cerebral sino también el conjunto de experiencias personales repetitivas, el género del ser humano y las sustancias corporales, quizá podemos comprender de manera más clara por qué en nuestra sociedad actual se observa un crecimiento de conductas antisociales y desinterés por la formación educativa.

Para lograr un cambio, se debe desarrollar programas específicos en menores que se encuentran en edad formativa (escolar) para contrarrestar la influencia o predisposición a generar conductas antisociales.

Entre ellas podríamos sugerir actividades que conlleven al desarrollo del Pensamiento Cognitivo Ejecutivo, el cual genera una mayor capacidad de planificar a largo plazo, de tener memoria de trabajo, además de jerarquizar ideas, establecer planes estratégicos y metas, ser flexibles con la adquisición de nuevos conocimientos $\mathrm{y}$, sobre todo, conseguir la atención selectiva voluntaria; con este gran potencial se podrá adquirir la capacidad de dirigir nuestra atención, con el fin de adquirir nuevos conocimientos y disminuir la predisposición a desarrollar una conducta violenta y disocial que se genera en el adolescente. La atención selectiva se ve acrecentada, principalmente, por la curiosidad o el entusiasmo que nos genere un aprendizaje, es importante encontrarle un sentido a lo que se estudia, y que no sólo se tome a la adquisición de conocimientos como un simple requisito para rendir un examen.

\section{CONCLUSIONES}

La neurociencia aparece hoy en día como un conjunto de disciplinas que mediante el estudio del funcionamiento de las bases del cerebro, nos permiten conocer las diversas actitudes, comportamientos y reacciones que genera el ser humano durante las diversas situaciones que se producen a lo largo de su existencia.

Una rama que se abre de estas disciplinas es la neuroeducación, a través de la cual, los docentes y entendidos en la materia, reconocen que las formas educativas actuales no son las más 
adecuadas para lograr el aprendizaje esperado, por el contrario, se ha visto incrementado el desinterés de los estudiantes de aprender y en mucho de los casos ha influenciado en la aparición de conductas indeseadas y criticadas por una sociedad que poco a poco está desquebrajándose y está siendo dominada por la violencia. Esto quizás se deba a la falta de estrategias que atraen el interés del estudiantado.

Para evitar que los menores que se encuentran en edad de formación presenten trastorno personal antisocial, especialmente los que manifiestan mayor predisposición a ello y que en mayor porcentaje se produce en el género masculino, y más bien desarrollen capacidades que contribuyan en su aprendizaje, es necesario desarrollar cambios en los programas educativos, los cuales deben estar inmersos en el conocimiento neurocientífico para así lograr un mejor desenvolvimiento del pensamiento cognitivo ejecutivo, mediante la comprensión de nuevos aprendizajes los cuales deben ser significativos. Para ello se debe desarrollar trabajos de asociación en las aulas ya que son una herramienta de gran ayuda en la educación y contribuyen a una mejor adquisición del aprendizaje; es por esto, que se deben aplicar estrategias que contribuyan a generar emociones significativas en el estudiante, porque lo ideal es buscar un sentido a lo que se estudia y relacionarlo con situaciones que beneficien nuestra vida. Esto sólo se puede dar a través del entendimiento del funcionamiento cerebral por parte del docente y que mediante estos conocimientos se pueda realizar una mejor forma de abordaje educativo, la que se necesita para lograr activar los órganos que forman parte del proceso de aprendizaje.

\section{REFERENCIAS}

Campos, L. (2010). Neuroeducación: uniendo las neurociencias y la educación en la búsqueda del desarrollo humano. Revista Digital:LaEducación.

Castro, M. (2015). La curiosidad contribuye con el aprendizaje y la memorización. Buenos Aires: Asociación Educar.

Damasio, A. (2006). El error de Descartes: emoción, razóny cerebrohumano. Editorial Crítica.

Del Ben, C. (2005). Neurobiología del trastorno de personalidadpersonal. Brasil.

Erk, S. et al. (2003). Emotional context modulates subsequent memory effect. Neuroimage, 18.
Garzón, Á. M. \& Sánchez, J. A. (2007, 02 de marzo). Factores neurobiológicos del trastorno de personalidad antisocial. RevistaPsicologiaCientifica.com, 9 (12).

Gonzales, G. T.; Campos, G. R.; Uribe, C. S. (2015). Neurociencia, contribución a la Historia. Bogotá: Instituto Nacional de la Salud.

Lino do Amaral, J. (2014). A contribuição da neurociência na formação de profesores. Río de Janeiro.

Logatt, C. (2014). Cerebro y aprendizaje: La atención selectiva. Argentina: Asociación Educar.

O'Rahilly, R. (1989). Anatomía de Gardner. 5ta. edición. México. 